(C) 2021 Universidad Nacional Autónoma de México, Facultad de Estudios Superiores Zaragoza.

Este es un artículo Open Access bajo la licencia CC BY-NC-ND (http://creativecommons.org/licenses/by-nc-nd/4.0/).

TIP Revista Especializada en Ciencias Químico-Biológicas, 24: 1-7, 2021.

https://doi.org/10.22201/fesz.23958723e.2021.341

\title{
2-Oxazolina: Polimerización y síntesis de macromonómeros
}

\author{
Michael Azael Ludeña-Huaman \\ Depto. Académico de Química, Facultad de Ciencias, Universidad \\ Nacional de San Antonio Abad del Cusco (UNSAAC), Av. De la \\ Cultura \# 733, Cusco, Perú. E-mail: michael.ludenah@unsaac.edu.pe
}

\begin{abstract}
RESUMEN
En las dos últimas décadas ha resurgido la química de la poli(2-oxazolina) debido a su fácil síntesis y capacidad para modular su estructura química, así como sus propiedades. Además, las poli(2-oxazolina)s son biocompatibles y muchas responden a un cambio de la temperatura. Los macromonómeros de 2-oxazolina usualmente son empleados como material de partida para realizar la síntesis a medida de copolímeros injertados, copolímeros de tipo estrella y dendrímeros. También los macromonómeros son introducidos en la síntesis de materiales con estructuras más complejas como los hidrogeles o nanogeles. Es motivo por el que, en este manuscrito se dan a conocer aspectos químicos fundamentales e importantes sobre la polimerización y preparación de los macromonómeros de 2-oxazolina, por el método del iniciado y terminador de la polimerización viviente. Se analiza la importancia del iniciador, el terminador y del sustituyente en la 2-oxazolina.
\end{abstract}

Palabras clave: 2-Oxazolina, macromonómero, polimerización, iniciador, terminador.

\section{2-Oxazoline: Polymerization and synthesis of macromonomers}

\begin{abstract}
The chemistry of poly(2-oxazoline) has resurfaced in the last two decades due to their easy synthesis, as well as the ability to modulate their chemical structure and properties. Furthermore, poly(2-oxazoline)s are biocompatible and many have thermoresponsive properties. 2-Oxazoline macromonomers are usually used as a starting material to perform the synthesis of graft copolymers, star copolymers and also dendrimers. But also macromonomers can be introduced in the synthesis of materials with more complex structures as hydrogels or nanogeles. For this reason, this manuscript describes fundamental and important chemical aspects about the polymerization and preparation of macromonomers by the initiator and terminator method of the living polymerization of 2-oxazoline. The importance of the initiator, terminator as well as the substituent on 2-oxazoline is discussed.
\end{abstract}

Keywords: 2-oxazoline, macromonomers, polymerization, initiator, terminator. 


\section{INTRODUCCIÓN}

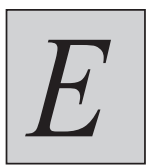

1 término macromonómero, se refiere a un monómero macromolecular o también a un polímero de bajo peso molecular con un grupo polimerizable al final de la cadena. En muchos casos el grupo polimerizable es el grupo vinilo, pero también puede ser el anillo oxirano o algún otro grupo capaz de polimerizar. Los macromonómeros que poseen algún grupo polimerizable en ambos extremos de la cadena son conocidos como telequélicos (Rempp \& Franta, 1984). El interés en la síntesis de macromonómeros, se debe a que por medio de estos compuestos se logra realizar de manera fácil la síntesis a medida de copolímeros injertados, copolímeros del tipo estrella y también dendrímeros (Schlüter \& Rabe, 2000) (Vazaios, Lohse \& Hadjichristidis, 2005). Del mismo modo, los macromonómeros son introducidos en la síntesis de materiales interesantes por su potencial aplicación en el campo de la biomedicina, como los hidrogeles (Kelly \& Wiesbrock, 2012) (Dargaville, Park \& Hoogenboom, 2018). Los métodos para realizar la síntesis permiten obtener macromonómeros bien definidos y funcionalizados (Ramírez, 2020). Estos métodos son clasificados como: a) la reacción de acoplamiento de un polímero preformado, b) la estrategia del iniciador y terminador de una polimerización viviente y c) la polimerización radicalaria por transferencia (Hadjichristidis, Pitsikalis, Latrou \& Pispas, 2003) (Patton \& Advincula, 2006).

Las 2-oxazolinas polimerizan vía catiónica por apertura del anillo, lo cual es una ventaja debido a que permite controlar el grado de polimerización. Además, varias poli(2-oxazolina)s responden al cambio de temperatura con mejor reversibilidad que la poli(N-isopropilacrilamida). Hoy en día, una cantidad significativa de investigaciones han sido dedicadas al uso de la poli(2-oxazolina) en la biomedicina, porque son compuestos no tóxicos y algunos de ellos son aprobados por la Administración de Medicamentos y Alimentos (FDA, por sus siglas en inglés); (Hoogenboom, 2009) (De la Rosa, 2014). En este manuscrito se aborda la preparación de los macromonómeros de 2-oxazolina, por el método del iniciador y terminador.

\section{Polimerización de 2-oxazolina}

La síntesis de las poli(2-oxazolina)s ocurren vía polimerización catiónica por apertura del anillo de las 2-oxazolinas, y tiene

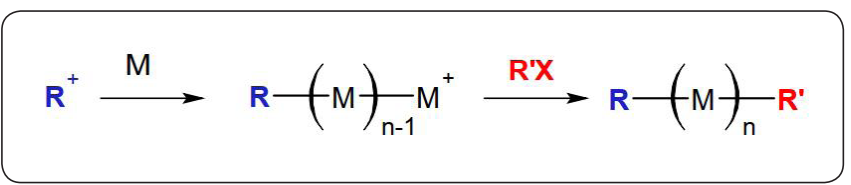

Figura 1. Reacción general dela formación de macromonómeros. Si $\mathbf{R}^{+}$es la molécula que contiene el grupo funcional polimerizable, entonces es referido como el método del iniciador y si R' es la molécula que contiene el grupo funcional polimerizable, entonces es referido como el método del terminador. Elaboración personal. un carácter "viviente" que puede ser aprovechado para ser funcionalizado. Las reacciones de polimerización ocurren en tres etapas: la iniciación, propagación y terminación. Para obtener el macromonómero de 2-oxazolina por el método del iniciador o terminador, se debe introducir el grupo polimerizable en la etapa de iniciación o de terminación de la polimerización de la 2-oxazolina (Figura 1) (Yamashita, 1993)

¿Qué es un polímero vivo?

Es un polímero que mantiene un sitio activo después de haber completado la etapa de reacción de propagación. Este sitio activo es aprovechado para funcionalizar el polímero o para continuar la polimerización con un monómero diferente y obtener un copolímero.

\section{MECANiSMo de POLIMERIZACIÓn de 2-OXAZOLINA}

La polimerización catiónica por apertura del anillo de 2-oxazolina inicia por la adición de un electrófilo, que es atacado por los electrones libres del nitrógeno de la 2-oxazolina y se genera una carga positiva sobre el nitrógeno que es estabilizado por resonancia. El siguiente paso es la propagación, y consiste en el ataque nucleofílico del nitrógeno de la 2-oxazolina hacia el carbono en posición cinco, que es el más electrófilo del híbrido de resonancia. Finalmente, la reacción culmina con la adición de un terminador nucleofílico (Figura 2).

La polimerización de la 2-oxazolina también podría seguir un mecanismo covalente como se muestra en la Figura 3.

El mecanismo de polimerización de la 2-oxazolina depende mucho de la nucleofilicidad del contra ión generado por el iniciador, otros aspectos que influyen pero en menor medida son los grupos sustituyentes en la 2-oxazolina y el solvente en el que se realiza la polimerización. Por esta razón, es importante elegir un iniciador adecuado, ya sea para favorecer un mecanismo de polimerización covalente o iónico. Por ejemplo, si se emplea el yoduro de bencilo como iniciador de la polimerización de 2-fenil2-oxazolina y 2-etil-2-oxazolina, la reacción de propagación de la 2-etil-2-oxazolina es prácticamente vía catiónica y de la 2-fenil-2-oxazolina es una mezcla entre la catiónica y la covalente. Sin embargo, si se emplea como iniciador el tosilato de bencilo la reacción de propagación en ambas 2-oxazolinas es completamente iónica (Fijten, Hoogenboom \& Schubert, 2008).

Los iniciadores frecuentemente usados en la polimerización de la 2-oxazolina son los triflatos, debido a que son electrófilos fuertes que aseguran un mecanismo netamente catiónico, cuya rapidez de iniciación $\left(\mathbf{K}_{\mathbf{i}}\right)$ es mucho mayor a la de la propagación $\left(\mathbf{K}_{\mathbf{P}}\right)$. El hecho de que $\mathbf{K}_{\mathbf{i}}$ sea mucho mayor que $\mathbf{K}_{\mathbf{P}}$, permite que la iniciación de la reacción sea simultánea y se minimice completamente la polidispersión(Luxenhofer, Bezen \& Jordan, 2008) (Glassner et al., 2015). Sin embargo, no se debe dejar de lado la nucleofilicidad de la 2-oxazolina, ya que también juega un rol importante entre el equilibrio catiónico y covalente. Los 


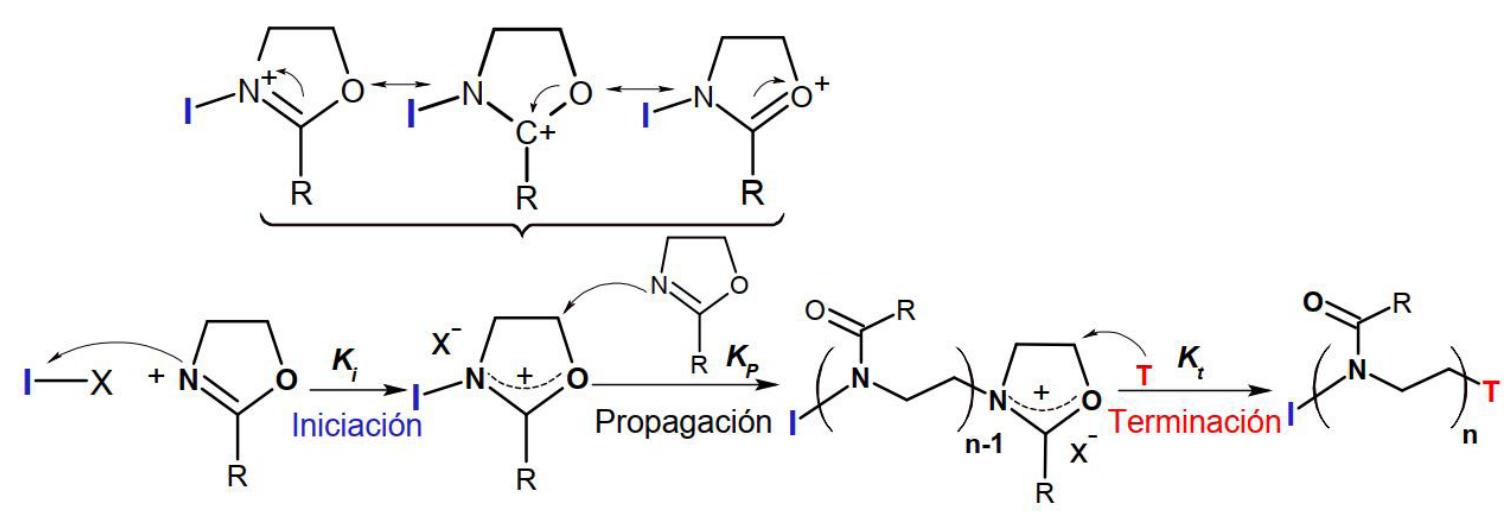

Figura 2. Mecanismo de reacción para la polimerización catiónica por apertura del anillo de 2-oxazolina. Elaboración personal.

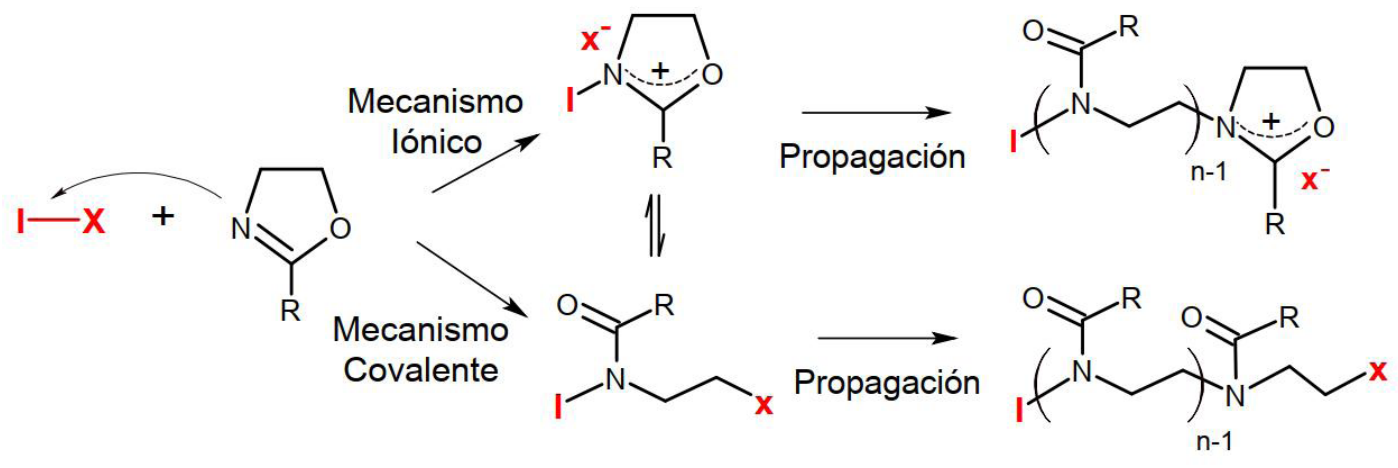

Figura 3. Mecanismo iónico y covalente de la polimerización de la 2-oxazolina. Elaboración personal.

sustituyentes en la 2-oxazolina originan una variación en la electrofilia del carbono en posición cinco y en la nucleofilia de la imina. Cuando el sustituyente es donante de electrones la nucleofilia de la imina incrementa $\left(\mathbf{K}_{\mathbf{i}}\right.$ incrementa) y es más susceptible a electrófilos, pero al mismo tiempo la electrofilia del carbono en posición cinco disminuye $\left(\mathbf{K}_{\mathbf{P}}\right.$ disminuye) y es menos susceptible a los nucleófilos. Estos efectos en la nucleofilia y electrofilia son inversos cuando el sustituyente es un atractor de electrones (Figura 4) (Kobayashi, Tokuzawa \& Saegusa, 1982). Cabe señalar que la rapidez de reacción de la polimerización está determinada por la rapidez de propagación $\mathbf{K}_{\mathbf{p}}$, por lo tanto si $\mathbf{K}_{\mathbf{p}}$ disminuye, entonces también la rapidez de reacción de la polimerización disminuye, y si $\mathbf{K}_{\mathbf{p}}$ aumenta, la rapidez de polimerización será mayor.

Cuando la polimerización se realiza con diferentes monómeros de 2-oxazolina, se obtienen copolímeros estadísticos o copolímeros en bloque. Los copolímeros estadísticos son formados mediante polimerización en un recipiente (traducido del inglés one-pot polymerization), la secuencia del monómero es dictado por la razón de reactividad $\mathrm{r}_{1} \mathrm{y} \mathrm{r}_{2}$. Si $\mathrm{r}_{1} \approx \mathrm{r}_{2} \approx 1$,

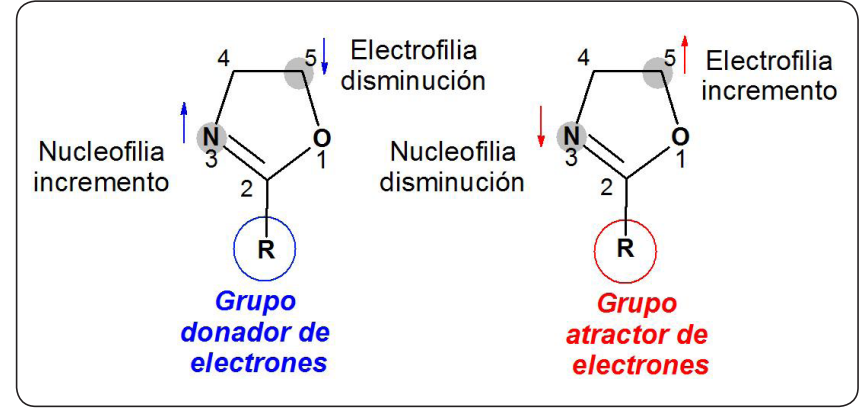

Figura 4. Efecto del grupo donador y atractor de electrones sobre la nucleofilia de la imina y la electrofilia del carbono en posición cinco. Elaboración personal.

esto significa que la reactividad de ambos monómeros son muy similares y el resultado es un verdadero copolímero aleatorio. Sin embargo, si uno es significativamente menor a 1 y el otro mayor a 1 , el polímero tendrá unidades repetidas del mismo monómero. Por otro lado, en lugar de polimerizar dos monómeros al mismo tiempo se podría realizar la 
polimerización primero de un monómero, y aprovechar su carácter "viviente" para añadir el segundo monómero sobre el primer monómero polimerizado, es así como se preparan los copolímeros en bloque. Se obtiene mejor resultado cuando el monómero más rápido se polimeriza primero, de esa forma se asegura que todas las cadenas reinicien la polimerización rápidamente después de adicionar el segundo monómero, ésta es una forma de cómo se pueden preparar copolímeros tribloques o di-bloques. La otra alternativa menos empleada es realizar una mezcla de ambos monómeros al mismo tiempo, a una temperatura determinada empieza a polimerizar uno de los monómeros, luego se incrementa la temperatura y empieza a polimerizar el otro monómero. Sin embargo, para realizar este procedimiento se requiere que los monómeros posean alta diferencia de reactividad (Verbraeken, Monnery, Lava \& Hoogenboom, 2017).

La reacción de terminación es fundamental al igual que la iniciación y la propagación. Los agentes terminadores suaves como el agua, tienen la tendencia de terminar sobre el carbono en posición dos y el resultado es la formación de una amina secundaria y un éster. Los agentes terminadores fuertes como los hidróxidos, carboxilatos y bases nitrogenadas terminan sobre el carbono en posición cinco. Se ha realizado un estudio sistemático sobre la rapidez de terminación, y se ha demostrado que existe una relación entre el valor de pka del agente terminador y la rapidez de terminación. El tiempo para culminar la reacción de terminación es menor cuando el valor de pka es mayor, puede tardar solo $10 \mathrm{~min}$ cuando se usa piperidina o soluciones etanólicas de $\mathrm{KOH}$, sin embargo, puede tardar hasta 22 horas cuando se usa piridina (Nuyken, Maier, GroB \& Fischer, 1996).

\section{Poli(2-Oxazolina)s Termosensibles}

Algunas poli(2-oxazolina)s son sensibles al cambio de temperatura, es decir experimentan una transición de solubilidad cuando ocurren cambios en la temperatura, y se produce una separación involuntaria de la poli(2-oxazolina) de la mezcla y aparece una inhomogeneidad. Existen dos tipos diferentes de polímeros termosensibles: los que sufren la transición de fase cuando se aumenta la temperatura, conocidos como polímeros de temperatura de solución crítica más baja LCST (por sus siglas en inglés, Lower Critical Solution Temperature), y los que sufren la transición cuando se disminuye la temperatura, conocidos como polímeros de temperatura de solución crítica más alta UCST (por sus siglas en inglés, Upper Critical Solution Temperature). Algunas poli(2-oxazolina)s termosensibles que han sido estudiadas son: las poli 2-etil-2-oxazolina $\mathrm{p}(\mathrm{EtOxa})$, poli 2-propil-2-oxazolina p(PrOxa), poli 2-ciclopropil-2-oxazolina $\mathrm{p}(\mathrm{CprOxa})$ y poli 2-isopropil-2-oxazolina $\mathrm{p}(\mathrm{IprOxa})$, etcétera (Figura 5) (Hoogenboom \& Schlaad, 2017).

La temperatura del punto nube $\left(T_{c p}\right)$, es la temperatura de separación de fase en una determinada concentración de polímero termosensible y se determina por turbidimetría. Los polímeros que muestran un comportamiento LCST son solubles en agua por debajo de su temperatura de transición, debido a la hidratación vía puente de hidrógeno entre el polímero-agua-polímero. A medida que se incrementa la temperatura, el enlace puente de hidrógeno se debilita, y se produce una deshidratación y mayor interacción entre polímero-polímero. Esto produce un colapso hidrofóbico y la formación de agregados (Bloksma et al., 2011) (Li, Tang \& Wu, 2015) (Figura 6).

La variación de $T_{c p}$ está determinada por factores como: a) Balance hidrofillico e hidrofóbico: se pueden realizar diferentes sustituciones en los monómeros de 2-oxazolina y modular el balance entre el carácter hidrofílico e hidrofóbico, por ejemplo, la poli(2-metil-2-oxazolina) que es bastante hidrofílica (soluble en agua) y la poli(2-butil-2-oxazolina) hidrofóbica (insoluble al agua), sin embargo, se podría modular el balance hidrofílico e hidrofóbico al usar un sustituyente intermedio, como en el caso de la poli(2-etil-2-oxazolina) o realizar un copolímero como poli(2-metil-2-oxazolina-co-2-butil-2-oxazolina) para que sea termosensible; b) Peso molecular: la generalidad es que a mayor peso molecular el $T_{c p}$ disminuye; c) Concentración: cuanto mayor sea la concentración el $T_{c p}$ disminuye y d) Los grupos terminales de la cadena: este es un factor que empieza a tomar relevancia en polímeros de bajo peso molecular (Lin, Clash, Pearce, Kwei \& Aponte, 1988) (Hoogenboom et al., 2008) (Obeid, Tanaka \& Winnik, 2009).

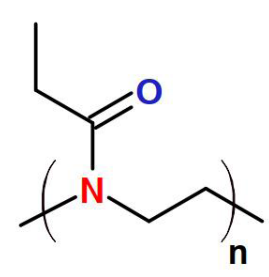

p(EtOxa)

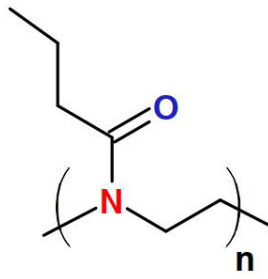

p(PrOxa)

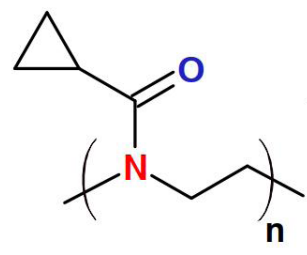

p(CprOxa)<smiles>CC(C)C(=O)N(CCC(C)(C)C)C(C)(C)C</smiles>

p(IprOxa)

Figura 5. Homopolímeros termosensibles de poli(2-oxazolina). Elaboración personal. 


\section{SÍNTESIS DE MACROMONÓMEROS DE 2-OXAZOLINA}

El enorme potencial de la 2-oxazolina se debe a su diversidad estructural y a la facilidad con la que se pueden modular sus propiedades. El carácter viviente de la polimerización por apertura del anillo permite la introducción de numerosos grupos funcionales a través del agente iniciador o terminador. La ventaja de usar un agente iniciador con grupo polimerizable, se debe a que deja libre la terminación para poder introducir otro grupo funcional. Sin embargo, se requiere que la velocidad de iniciación sea mayor a la de propagación, para evitar transferencias de cadena, muchas veces esto es difícil de conseguir con agentes iniciadores con grupo polimerizable. Esta es la razón, de por qué la introducción de un grupo polimerizable en la reacción de terminación es más recomendable, el principal requisito es que el agente terminador sea un nucleófilo fuerte, reaccione rápidamente e irreversiblemente con el oxazolinio. En la Figura 7 se muestra el mecanismo de la polimerización viviente por apertura del anillo de las 2-oxazolinas, también algunos agentes iniciadores y terminadores que han sido usados para la síntesis del macromonómero (Pizzi et al., 2019).

\section{Conclusiones}

En este trabajo se describe el mecanismo de polimerización viviente catiónica por apertura del anillo de 2-oxazolina y la preparación de macromonómeros. La electrofilicidad del iniciador es un factor importante, que determina el mecanismo de polimerización y la polidispersidad. La nucleofilicidad del terminador determina la rapidez de terminación y tiene relación directa con el valor de pKa. Los sustituyentes atractores de electrones en la 2-oxazolina incrementan la rapidez de la polimerización, los sustituyentes donadores de electrones causan el efecto contrario. El método de preparación de macromonómeros que se aborda es del iniciador y terminador, la introducción del grupo polimerizable en la terminación
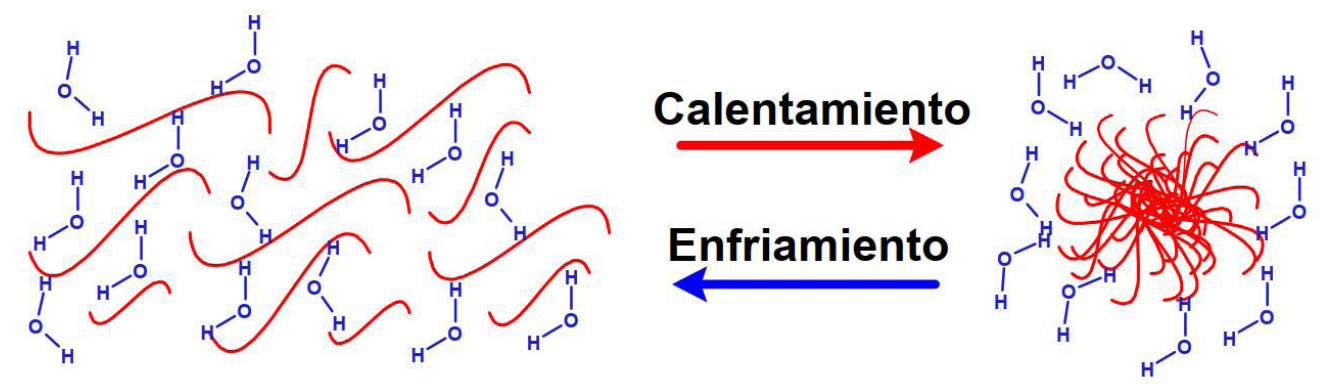

Figura 6. Representación esquemática de la transición de solubilidad de los polímeros termosensibles (LCST). Hidratación de las cadenas poliméricas vía puente de hidrógeno (izquierda) y formación del agregado hidrofóbico (derecha). Elaboración personal.

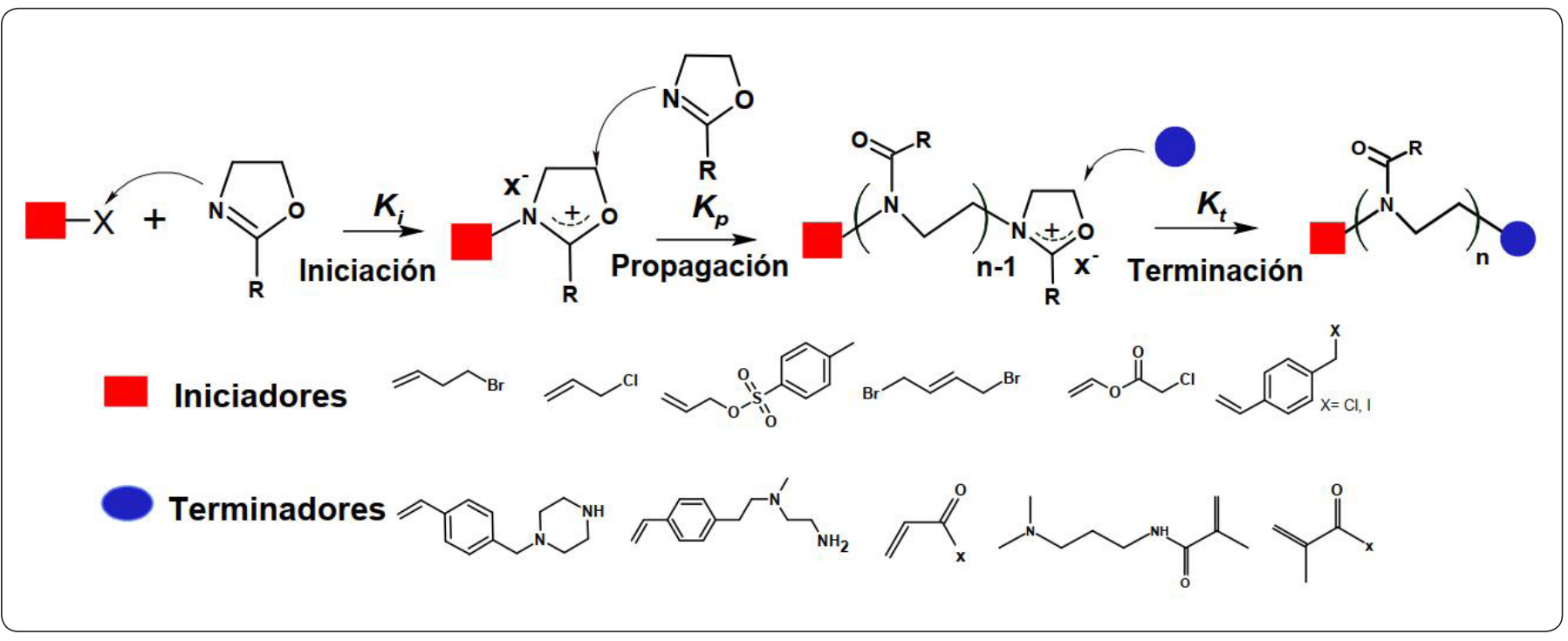

Figura 7. Mecanismo de reacción de la formación de macromonómeros vía agente iniciador $(\square)$ o terminador $(O)$ para la polimerización catiónica por apertura del anillo de 2-oxazolina. - Iniciadores usualmente empleados; terminadores usualmente empleados. Elaboración personal. 
es más recomendable. Se presentan algunos de los agentes iniciador y terminador más utilizados en la preparación de los macromonómeros. También se realiza una descripción de los aspectos fundamentales que influyen en la respuesta al cambio de la temperatura de las poli(2-oxazolina)s. Se desea que el manuscrito sea de ayuda complementaria para quienes aborden el estudio de la polimerización y preparación de macromonómeros de 2-oxazolina.

\section{Agradecimientos}

Agradezco a la Prof. Janet González Bellido y Prof. Ana María Lechuga Chacón por el constante apoyo que me brindan y también a los revisores por las sugerencias realizadas para mejorar el manuscrito.

\section{CONFLiCTO DE INTERESES}

No existe conflicto de intereses.

\section{REFERENCIAS}

Bloksma, M. M., Weber, C., Perevyazko, I. Y., Kuse, A., Baumgärtel, A., Vollrath, A., Hoogenboom, R. \& Schubert, U. S. (2011). Poly(2-cyclopropyl-2-oxazoline): From Rate Acceleration by Cyclopropyl to Thermoresponsive Properties. Macromolecules, 44(11), 4057-4064. https:// doi.org/10.1021/ma200514n

Dargaville, T. R., Park, J. R. \& Hoogenboom, R. (2018). Poly(2oxazoline) Hydrogels: State-of-the-Art and Emerging Applications. Macromolecular Bioscience, 18(6), 1800070. https://doi.org/10.1002/mabi.201800070

De la Rosa, V. R. (2014). Poly(2-oxazoline)s as materials for biomedical applications. Journal of Materials Science: Materials in Medicine, 25(5), 1211-1225. https://doi. org/10.1007/s10856-013-5034-y

Fijten, M. W. M., Hoogenboom, R. \& Schubert, U. S. (2008). Initiator effect on the cationic ring-opening copolymerization of 2-ethyl-2-oxazoline and 2-phenyl-2-oxazoline. Journal of Polymer Science Part A: Polymer Chemistry, 46(14), 4804-4816. https://doi.org/10.1002/pola.22814

Glassner, M., D’hooge, D. R., Young Park, J., Van Steenberge, P. H. M., Monnery, B. D., Reyniers, M.-F. \& Hoogenboom, R. (2015). Systematic investigation of alkyl sulfonate initiators for the cationic ring-opening polymerization of 2-oxazolines revealing optimal combinations of monomers and initiators. European Polymer Journal, 65, 298-304. https://doi.org/10.1016/j. eurpolymj.2015.01.019

Hadjichristidis, N., Pitsikalis, M., Iatrou, H. \& Pispas, S. (2003). The Strength of the Macromonomer Strategy for Complex Macromolecular Architecture: Molecular Characterization, Properties and Applications of Polymacromonomers. Macromolecular Rapid Communications, 24(17), 9791013. https://doi.org/10.1002/marc.200300050

Hoogenboom, R. (2009). Poly(2-oxazoline)s: A Polymer Class with Numerous Potential Applications. Angewandte Chemie
International Edition, 48(43), 7978-7994. https://doi. org/10.1002/anie.200901607

Hoogenboom, R. \& Schlaad, H. (2017). Thermoresponsive poly(2-oxazoline)s, polypeptoids, and polypeptides. Polymer Chemistry, 8(1), 24-40. https://doi.org/10.1039/ C6PY01320A

Hoogenboom, R., Thijs, H. M. L., Jochems, M. J. H. C., Lankvelt, B. M. van, Fijten, M. W. M. \& Schubert, U. S. (2008). Tuning the LCST of poly(2-oxazoline)s by varying composition and molecular weight: Alternatives to poly $(\mathrm{N}-$ isopropylacrylamide)?. Chemical Communications, 44, 5758-5760. https://doi.org/10.1039/B813140F

Kelly,A. M. \& Wiesbrock, F. (2012). Strategies for the Synthesis of Poly(2-Oxazoline)-Based Hydrogels. Macromolecular Rapid Communications, 33(19), 1632-1647. https://doi. org/10.1002/marc.201200333

Kobayashi, S., Tokuzawa, T. \& Saegusa, T.(1982). Cationic ringopening isomerization polymerization of $2-[\mathrm{p}-($ substituted) phenyl]-2-oxazolines. Effects of the substituent on the reactivities. Macromolecules, 15(6), 707-710. https://doi. org/10.1021/ma00231a005

Li, T., Tang, H. \& Wu, P. (2015). Molecular Evolution of Poly(2-isopropyl-2-oxazoline) Aqueous Solution during the Liquid-Liquid Phase Separation and Phase Transition Process. Langmuir, 31(24), 6870-6878. https://doi. org/10.1021/acs.langmuir.5b01009

Lin, P., Clash, C., Pearce, E. M., Kwei, T. K. \& Aponte, M. A. (1988). Solubility and miscibility of poly(ethyl oxazoline). Journal of Polymer Science Part B: Polymer Physics, 26(3), 603-619. https://doi.org/10.1002/polb.1988.090260312

Luxenhofer, R., Bezen, M. \& Jordan, R. (2008). Kinetic Investigations on the Polymerization of 2-Oxazolines Using Pluritriflate Initators. Macromolecular Rapid Communications, 29(18), 1509-1513. https://doi. org/10.1002/marc.200800293

Nuyken, O., Maier, G., Groß,A. \& Fischer, H. (1996). Systematic investigations on the reactivity of oxazolinium salts. Macromolecular Chemistry and Physics, 197(1), 83-95. https://doi.org/10.1002/macp.1996.021970106

Obeid, R., Tanaka, F. \& Winnik, F. M. (2009). Heat-Induced Phase Transition and Crystallization of Hydrophobically End-Capped Poly(2-isopropyl-2-oxazoline)s in Water. Macromolecules, 42(15), 5818-5828. https://doi. org/10.1021/ma900838v

Patton, D. L. \& Advincula, R. C. (2006). A Versatile Synthetic Route to Macromonomers via RAFT Polymerization. Macromolecules, 39(25), 8674-8683. https://doi. org/10.1021/ma061382h

Pizzi, D., Humphries, J., Morrow, J. P., Fletcher, N. L., Bell, C. A., Thurecht, K. J. \& Kempe, K. (2019). Poly(2-oxazoline) macromonomers as building blocks for functional and biocompatible polymer architectures. European Polymer Journal, 121, 109258. https://doi.org/10.1016/j. eurpolymj.2019.109258 
Ramírez, J. M. C. (2020). Macromonómeros: Síntesis y Aplicaciones. Revista Bases de la Ciencia, 5(1), 15-40. https://doi.org/10.33936/rev_bas_de_la_ciencia. v5i1.1904

Rempp, P. F. \& Franta, E. (1984) Macromonomers: Synthesis, characterization and applications. In: Polymerization Reactions. Advances in Polymer Science, 58. Springer, Berlin, Heidelberg. https://doi.org/10.1007/3-540-127933 -6

Schlüter, A. D. \& Rabe, J. P. (2000). Dendronized Polymers: Synthesis, Characterization, Assembly at Interfaces, and Manipulation. Angewandte Chemie International Edition,
39(5), 864-883. https://doi.org/10.1002/(SICI)15213773(20000303)39:5<864::AID-ANIE864>3.0.CO;2-E

Vazaios, A., Lohse, D. J. \& Hadjichristidis, N. (2005). Linear and Star Block Copolymers of Styrenic Macromonomers by Anionic Polymerization. Macromolecules, 38(13), 5468-5474. https://doi.org/10.1021/ma0473364

Verbraeken, B., Monnery, B. D., Lava, K. \& Hoogenboom, R. (2017). The chemistry of poly(2-oxazoline)s. European Polymer Journal, 88,451-469. https://doi.org/10.1016/j. eurpolymj.2016.11.016

Yamashita, Y.(1993). Chemistry andindustryofmacromonomers. Hüthig \& Wepf Verlag. 